\title{
Electrochemistry of porous and crystalline silicon electrodes in methylviologen solutions
}

\author{
E.S. Kooij, R.W. Despo, F.P.J. Mulders, J.J. Kelly \\ Department of Condensed Matter, Debye Institute, Utrecht University, PO Box 80 000, 3508 TA Utrecht, Netherlands
}

Received 8 September 1995; in revised form 23 October 1995

\begin{abstract}
From measurements using stationary and rotating disc and ring-disc electrodes, it is concluded that the reduction reactions of the divalent methylviologen cation $\mathrm{MV}^{2+}$ (to $\mathrm{MV}^{+\cdot}$ and $\mathrm{MV}^{0}$ ) proceed via the conduction band of both porous and crystalline silicon. The product of the second reduction step $\left(\mathrm{MV}^{0}\right)$ forms a blocking layer on the electrode. The oxidation reactions of $\mathrm{MV}^{0}$ and $\mathrm{MV}^{+} \cdot$ take place by electron injection into the conduction band of n-type crystalline silicon. From the current-potential characteristics it follows that $\mathrm{MV}^{+\cdot}$ is also able to inject electrons into porous silicon formed on an n-type electrode. At p-type porous silicon, electroluminescence is observed as a result of radiative recombination of holes (majority carriers) with electrons (minority carriers) injected into the porous structure during oxidation of $\mathrm{MV}^{+}$. Introducing ethanol into the solution leads to changes in the current-potential characteristics, which are explained by the solubility of $\mathrm{MV}^{0}$ in these solutions. The current-potential characteristics are considered on the basis of the position of the band edges, as deduced from impedance measurements, and differences between crystalline and porous silicon are discussed.
\end{abstract}

Keywords: Methylviologen reduction; Porous silicon; Crystalline silicon; Current-potential characteristics; Electroluminescence

\section{Introduction}

Silicon is, despite its excellent electrical properties, not suitable for the fabrication of light emitting devices because of its indirect band gap. Since the first report of visible photoluminescence ( $\mathrm{PL}$ ) from porous silicon at room temperature [1] a few years ago, this material has attracted much attention. The main goal of the research is the development of an efficient all-silicon electroluminescent device. Measurements on porous silicon/liquid junctions have provided important insight into the properties of the porous material; the solution penetrates into the pores, forming a contact to the whole porous structure. This is in contrast to most solid state devices, where only the top of the porous structure is contacted [2-4]. Strong visible EL has been observed from n-type porous silicon in contact with a solution containing a strong oxidizing agent $[5,6]$ and the wavelength of the emission maximum is a function of the applied potential $[7,8]$.

Recently, we reported electroluminescence (EL) from p-type porous silicon electrodes in contact with a solution containing a reducing agent [9]. The injection of the minority carriers (electrons) was achieved by oxidation of the methylviologen (1,1'-dimethyl-4,4'-bipyridinium) radical cation $\mathrm{MV}^{+}$, which is formed in the first reduction step of the methylviologen cation $\mathrm{MV}^{2+}$

$\mathrm{MV}^{2+}+\mathrm{e}^{-} \rightleftharpoons \mathrm{MV}^{+}$

This reaction is highly reversible and has a redox potential of $-0.69 \mathrm{~V}$ vs. a saturated calomel electrode (SCE) [10]. Further reduction of $\mathrm{MV}^{+\cdot}$ is more complicated and depends on the local concentration of methylviologen near the electrode surface [11]. In its simplest form, the second reduction can be described by a reversible reaction

$\mathrm{MV}^{+\cdot}+\mathrm{e}^{-} \rightleftharpoons \mathrm{MV}^{0}$

Different values are reported for the redox potential of this reaction: $-1.1 \mathrm{~V}[10,12]$ and $-1.35 \mathrm{~V}$ vs. SCE [11]. The $\mathrm{MV}^{0}$ formed at the electrode surface is not soluble in aqueous solutions; it forms a solid film on the electrode [13]. The exact nature of the film is unclear; on gold electrodes a layer of $\mathrm{MV}^{0}$ is formed, while at an edge carbon electrode an $\mathrm{MV}^{0}-\mathrm{MV}^{+\cdot}$ mixed-valence film is reported.

In this paper the emphasis is on a comparison of the electrochemistry of methylviologen at porous and crystalline silicon electrodes. The EL intensity of $n$-type porous 
silicon is reported to be strongly enhanced by introducing ethanol into the solution [14-16]. It was assumed that the addition of ethanol facilitates the penetration of the solution into the hydrophobic porous structure [15]. To investigate if this is also the case with p-type electrodes, we worked with both aqueous and mixed ethanol + water solutions. We performed impedance measurements on crystalline and porous silicon electrodes to determine the position of the band edges. These measurements form the basis for an analysis of charge transfer reactions of methylviologen at crystalline and porous silicon electrodes.

\section{Experimental}

Electrodes were cut from n-type $(0.7$ to $0.9 \Omega \mathrm{cm})$ and p-type ( 8 to $12 \Omega \mathrm{cm}$ ) single crystal silicon wafers which had the $(100)$ orientation. Crystalline silicon electrodes were dipped in a $1 \mathrm{M} \mathrm{HF}+2 \mathrm{M} \mathrm{NH}_{4} \mathrm{~F}$ solution for $1 \mathrm{~min}$ before each measurement to remove surface oxides. Porous silicon electrodes were formed by anodic etching for $5 \mathrm{~min}$ at $35 \mathrm{~mA} \mathrm{~cm}{ }^{-2}$ in a 1:1 mixture of $20 \%$ HF:ethanol. A two-electrode configuration with a Pt counter electrode was used. Anodization was performed under illumination at $0.4 \mathrm{~V}$ cell voltage for n-type and galvanostatically in the dark for p-type. Before and after each measurement the presence of the luminescent porous layer was checked by a PL measurement at an excitation wavelength of $354 \mathrm{~nm}$.

The electrochemical experiments were performed using an EG\& G Princeton Applied Research (PAR) 366A bipotentiostat in a conventional electrochemical cell containing a $\mathrm{Au}$ or Pt counter electrode and an SCE as reference. All potentials are given with respect to SCE. For measurements using the rotating disc electrode (RDE) configuration the working electrode was a silicon disc $(4.0 \mathrm{~mm}$ diameter). For the rotating ring-disc electrode (RRDE) experiments we used a silicon ring $(4.55 \mathrm{~mm}$ and $6.9 \mathrm{~mm}$ inner and outer diameters respectively) as the working electrode. A Au disc ( $4.0 \mathrm{~mm}$ diameter) was used to generate active redox species. From these values a collection efficiency of 0.49 was calculated [17].

The impedance measurements were performed with an EG\&G PAR 273A potentiostat in combination with a Solartron HF frequency response analyser. EL was measured using a standard Si photodiode in combination with an Oriel amplifier (Model 70710). For illumination, a white light source (Schott KL 1500) was used. In all experiments involving illumination of p-type electrodes the photon flux was larger than the diffusion flux of $\mathrm{MV}^{2+}$ to the electrode.

The $\mathrm{MVCl}_{2}$ was obtained from Fluka. All other chemicals (Merck) were of p.a. quality. The $\mathrm{pH}$ of all solutions was between 6 and 7. Before each measurement, high purity Ar gas was bubbled through the solution to remove oxygen.

\section{Results}

\subsection{Current-potential measurements at stationary and RDES}

Current-potential curves of stationary $\mathrm{n}$ - and p-type crystalline silicon electrodes in an aqueous $2 \mathrm{mM} \mathrm{MVCl}{ }_{2}$ $+0.1 \mathrm{M} \mathrm{KCl}$ solution are shown in Figs. 1(a) and 1(b) respectively. Measurements on n-type silicon were performed in the dark, while the p-type electrode was studied in the dark and under illumination. In the dark, only a very small current is found with the p-type electrode (dashed line). When the potential of both n-type and illuminated p-type electrodes (solid curves) is scanned in the negative direction, two current maxima are observed. The first current peak can be ascribed to the first reduction step of $\mathrm{MV}^{2+}$. The redox potential of this reaction $(-0.69 \mathrm{~V})$ agrees with the potential at which current onset is found with n-type silicon, while with p-type silicon the current onset is shifted to $-0.3 \mathrm{~V}$. We assume the second current maximum to correspond to the second reduction step $\left(\mathrm{MV}^{+\cdot}\right.$ to $\left.\mathrm{MV}^{0}\right)$. The height of both maxima depends on the rate at which the potential is scanned, indicating that the reduction reactions are diffusion limited. At more negative potentials the current increases due to hydrogen evolution. In the return scan to positive potentials a difference is observed between the two types of electrode. With n-type silicon two peaks are again found. The first peak is

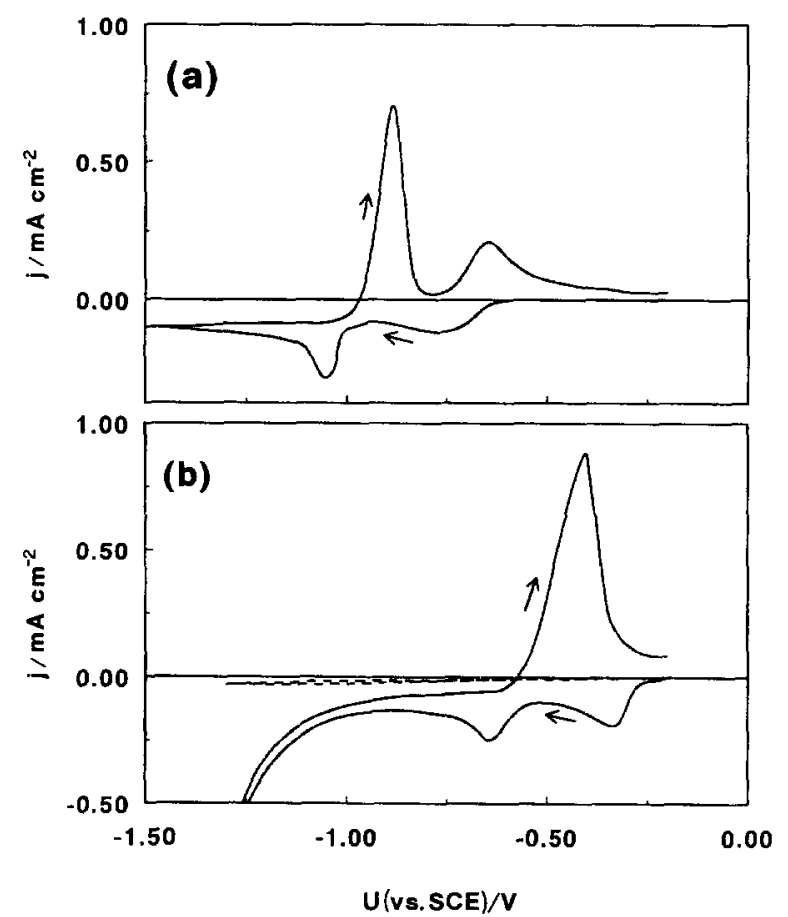

Fig. 1. Current-potential curves of stationary (a) n-type and (b) p-type crystalline silicon electrodes in $2 \mathrm{mM} \mathrm{MVCl}_{2}+0.1 \mathrm{M} \mathrm{KCl}$ aqueous solution. Measurements with the n-type electrode were made in the dark, while the p-type electrode was studied in the dark (dashed line) and under illumination (solid line). The potential was scanned at $10 \mathrm{mV} \mathrm{s}^{-1}$. 
much higher than the second and is related to the oxidation of $\mathrm{MV}^{0}$. As mentioned in the Introduction, $\mathrm{MV}^{0}$ forms a solid film on the electrode and is thus unable to diffuse away from the surface, which explains the larger anodic current in the first maximum. The second maximum at $-0.7 \mathrm{~V}$ results from reoxidation of $\mathrm{MV}^{+\cdot}$ to $\mathrm{MV}^{2+}$. Only a single peak is found with the p-type electrode, presumably due to an overlap of the two oxidation peaks.

From the fact that a cathodic current is found with n-type electrodes in the dark and with p-type electrodes under illumination, while only a very small dark current is detected with p-type silicon, we conclude that both reduction reactions (1) and (2) occur via electron capture from the conduction band. In the return scan towards positive potentials the observation of anodic current with n-type silicon in the dark shows that both oxidation reactions occur via electron injection, as there are no holes present at the electrode surface. Since, as will be shown, the band edges of n-type and p-type silicon are at the same position, it is likely that oxidation reactions of $\mathrm{MV}^{0}$ and $\mathrm{MV}^{+\cdot}$ at p-type silicon also take place via electron injection into the conduction band.

Figs. 2(a) and 2(b) respectively show the effect of electrode rotation on the current-potential curves obtained with n-type silicon in the dark and p-type silicon under illumination. The solution composition was similar to that used for the experiments of Fig. 1 and the rotation rate was $1600 \mathrm{rev} \min ^{-1}$. The onset of the cathodic current due to reduction of $\mathrm{MV}^{2+}$ occurs at the same potentials as in Fig. 1 , but the current reaches a limiting value, as is clear from the plateau in the current-potential curves. This plateau is followed by a cathodic peak, whose maximum strongly depends on the scan rate and the rotation rate. This peak is due to the formation of the solid film of $\mathrm{MV}^{0}$, mentioned earlier. At high scan rates and low rotation rates the peak broadens to give a second plateau; the current density in this plateau is twice that of the first plateau. At potentials more negative than the cathodic maximum this layer blocks further reduction of $\mathrm{MV}^{+}$, but the first reduction step can obviously still proceed as a plateau is again observed at the same level as the first plateau. When the potential is scanned towards positive values the current density remains constant until a sharp peak is found, which corresponds to oxidation of the solid $\mathrm{MV}^{0}$ layer. This is supported by the fact that the peak maximum and shape are almost independent of the range over which the potential is scanned, but the peak is absent when the scan direction is reversed before the onset of $\mathrm{MV}^{0}$ formation. After this peak the current again decreases to zero. The anodic current maximum owing to the oxidation of $\mathrm{MV}^{+}$. observed in Fig. 1 is absent when the electrode is rotated; $\mathrm{MV}^{+}$is not adsorbed at the surface and is thus swept away from the electrode during rotation.

In Fig. 2(c) the limiting current in the first plateau (at $-0.55 \mathrm{~V}$ for p-type silicon) is plotted as a function of the square root of the rotation rate. From the linear relation it
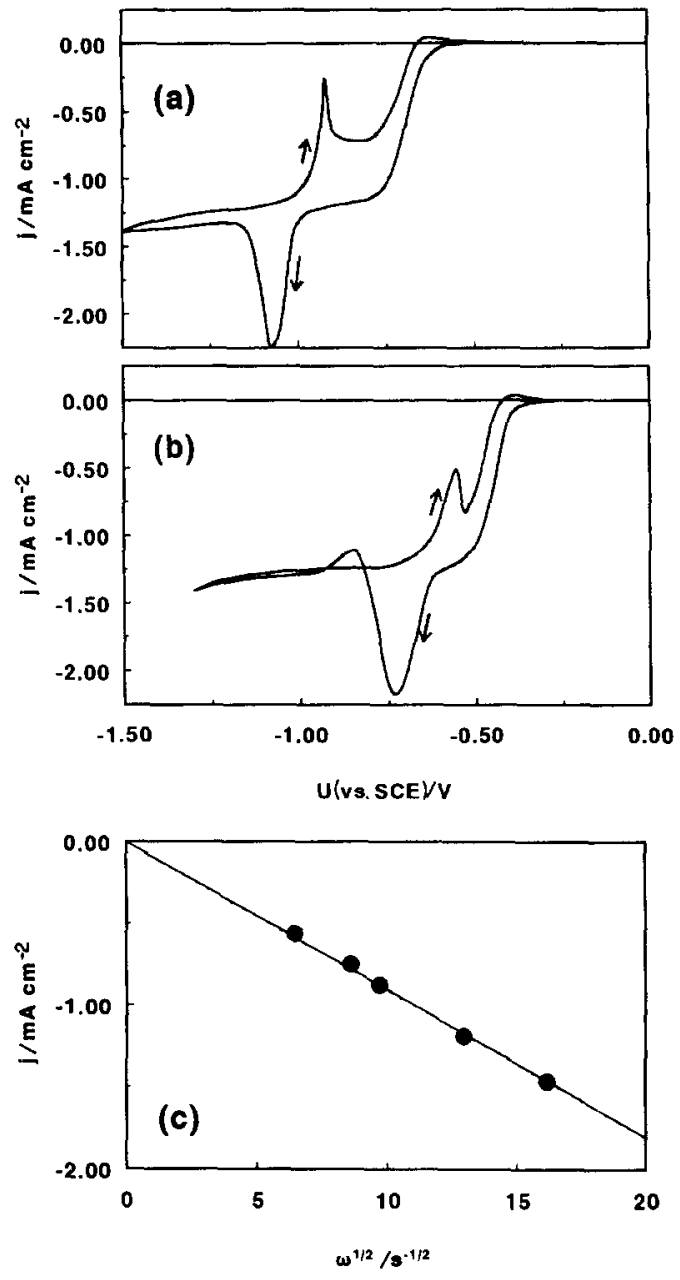

Fig. 2. Current-potential curves of (a) n-type and illuminated (b) p-type crystalline silicon electrodes in $2 \mathrm{mM} \mathrm{MVCl} 2+0.1 \mathrm{M} \mathrm{KCl}$ aqueous solution. The potential was scanned at $10 \mathrm{mV} \mathrm{s}^{-1}$, while the electrode was rotated at $1600 \mathrm{rev} \mathrm{min}^{-1}$. (c) The current density of the p-type electrode at $-0.55 \mathrm{~V}$ as a function of the square root of the rotation rate.

follows that the rate of the first reduction reaction is determined by mass transport. Using the Levich equation we calculate a diffusion coefficient of $6.5 \times 10^{-6} \mathrm{~cm}^{2} \mathrm{~s}^{-1}$ for the $\mathrm{MV}^{2+}$ species. This value is comparable with that reported in the literature [10].

In Fig. 3 current-potential curves of a stationary n-type porous silicon electrode are shown. The solution is similar to that used for the experiments of Figs. 1 and 2. When the potential is scanned to $-0.8 \mathrm{~V}$ and back (solid line) so that only $\mathrm{MV}^{+\cdot}$ is formed, the curves are reproducible. In the return scan an anodic peak is observed due to oxidation of $\mathrm{MV}^{+}$. The fact that this peak is present in the dark with n-type electrodes implies that $\mathrm{MV}^{+}$is also able to inject electrons into the conduction band of porous silicon electrodes. With p-type porous silicon the reduction of $\mathrm{MV}^{2+}$ to $\mathrm{MV}^{+}$only occurs when the electrode is illuminated, indicating that electrons from the conduction band are involved. When the potential is scanned into the region where $\mathrm{MV}^{0}$ is formed (dashed line in Fig. 3), the 


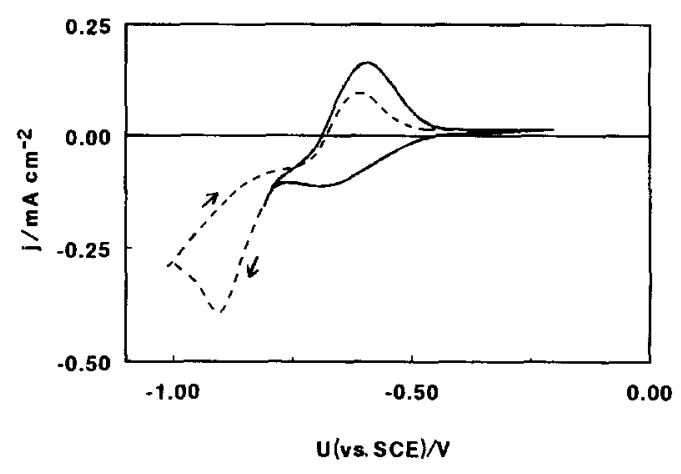

Fig. 3. Current-potential curves of a stationary n-type porous silicon electrode in $2 \mathrm{mM} \mathrm{MVCl} 2+0.1 \mathrm{M} \mathrm{KCl}$ aqueous solution. For the solid curve, the lower limit of the scan was $-0.8 \mathrm{~V}$. The dashed curve was scanned to $-1.0 \mathrm{~V}\left(10 \mathrm{mV} \mathrm{s}^{-1}\right)$.

current-potential curves are much less reproducible. From PL measurements we found that the luminescent porous layer is removed after a few scans. Apparently, growth of
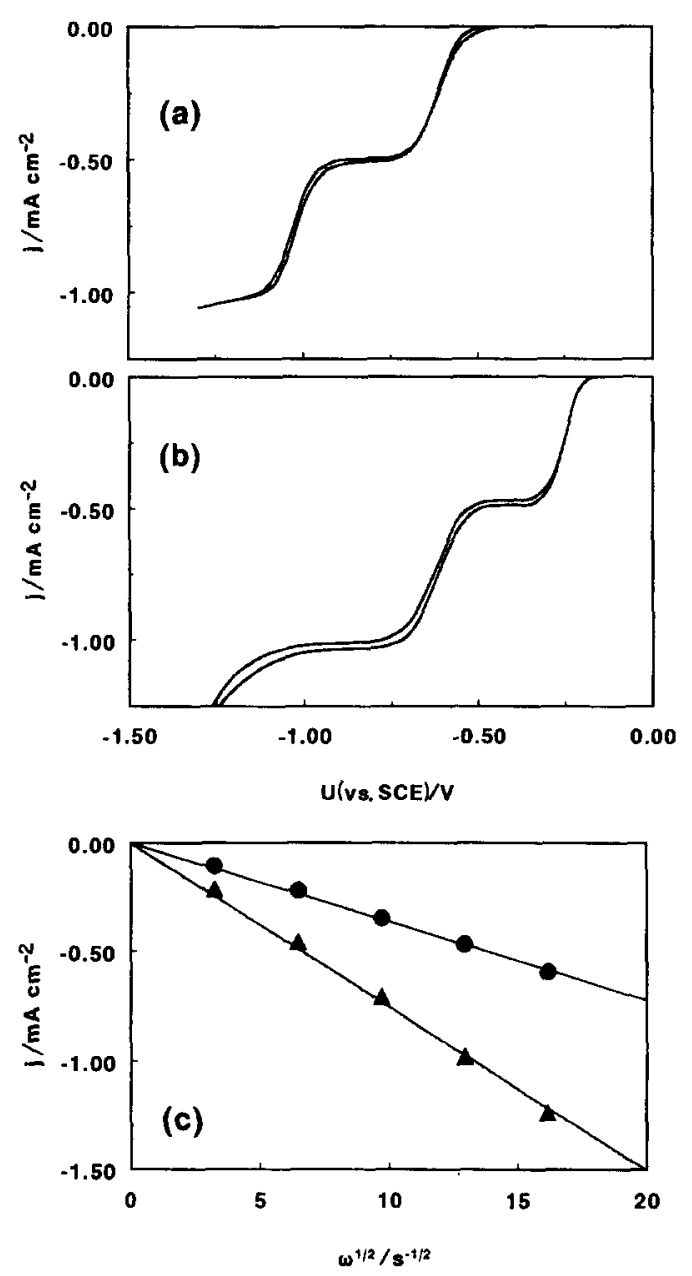

Fig. 4. Current-potential curves of (a) n-type and illuminated (b) p-type crystalline silicon electrodes in $2 \mathrm{mM} \mathrm{MVCl}{ }_{2}+0.1 \mathrm{M} \mathrm{KCl} \mathrm{1:1} \mathrm{water} \mathrm{+}$ ethanol solution. The potential was scanned at $10 \mathrm{mV} \mathrm{s}^{-1}$, while the electrode was rotated at $1600 \mathrm{rev} \mathrm{min}^{-1}$. (c) The current density of the p-type electrode at $-0.4 \mathrm{~V}$ (circles) and $-0.75 \mathrm{~V}$ (triangles) as a function of the square root of the rotation rate. the solid $\mathrm{MV}^{0}$ layer in the pores mechanically destroys the porous layer and, after one or two scans, only the crystalline substrate remains.

Measurements on methylviologen in a 1:1 mixture of water + ethanol yielded somewhat different results. Fig. 4 shows current-potential curves of (a) rotating n-type and (b) illuminated p-type electrodes in $2 \mathrm{mM} \mathrm{MVCl}_{2}+0.1 \mathrm{M}$ $\mathrm{KCl}$ water + ethanol solution. Again, a plateau due to the first reduction reaction of $\mathrm{MV}^{2+}$ is observed. The second reduction step no longer leads to a cathodic current peak, but now a second plateau is observed with a limiting current twice that of the first plateau. We assume that the $\mathrm{MV}^{0}$ formed in this step is soluble and does not form a layer on the electrode surface. This explains why an anodic peak is not observed in the return scan towards positive potentials. As for the previous measurements, we conclude that both reduction reactions proceed by electron capture from the conduction band, as only a very small dark current is found with p-type electrodes.

Fig. 4(c) shows the linear relation between the limiting current density for the two steps (at $-0.4 \mathrm{~V}$ and $-0.75 \mathrm{~V}$ for p-type silicon) and the square root of the rotation rate, indicating that in this solution both reactions are diffusion limited. However, the limiting current is considerably lower than that found in the previous measurements, which can be explained by a difference in viscosity of the water + ethanol mixture compared to that of pure water. The kinematic viscosity of a 1:1 mixture of water + ethanol at $20^{\circ} \mathrm{C}$ is 3.043 [18]. Using the Levich equation and assuming that $D \propto 1 / \nu[19]$, it follows that the limiting current should be $40 \%$ of that found in water, which is indeed observed in Fig. 4(c).

\subsection{Current-potential measurements at ring-disc elec- trodes}

As mentioned in the introduction, EL from p-type porous silicon can only be generated when electrons are injected into the conduction band of porous silicon. From the previous results we concluded that the electrochemical reactions of methylviologen proceed mainly by charge transfer to and from the conduction band. However, as $\mathrm{MV}^{0}$ destroys the porous structure, the only species capable of injecting electrons is the radical cation. To obtain a large flux of $\mathrm{MV}^{+}$, we used the RRDE configuration with a Au disc and a silicon ring. In Fig. 5(a) a potentiodynamic measurement in $2 \mathrm{mM} \mathrm{MVCl}+0.1 \mathrm{M} \mathrm{KCl}$ aqueous solution is shown. The disc potential was scanned, while the ring potential was kept at a constant value of $-0.2 \mathrm{~V}$. At the disc we see characteristics similar to those found before with the silicon RDE (Fig. 2), while at the ring we find an anodic current which strongly depends on processes occurring at the disc. In the first plateau the $\mathrm{MV}^{+}$. generated at the disc is oxidized at the ring. When the cathodic peak is reached at the disc, less $\mathrm{MV}^{+}$is detected 

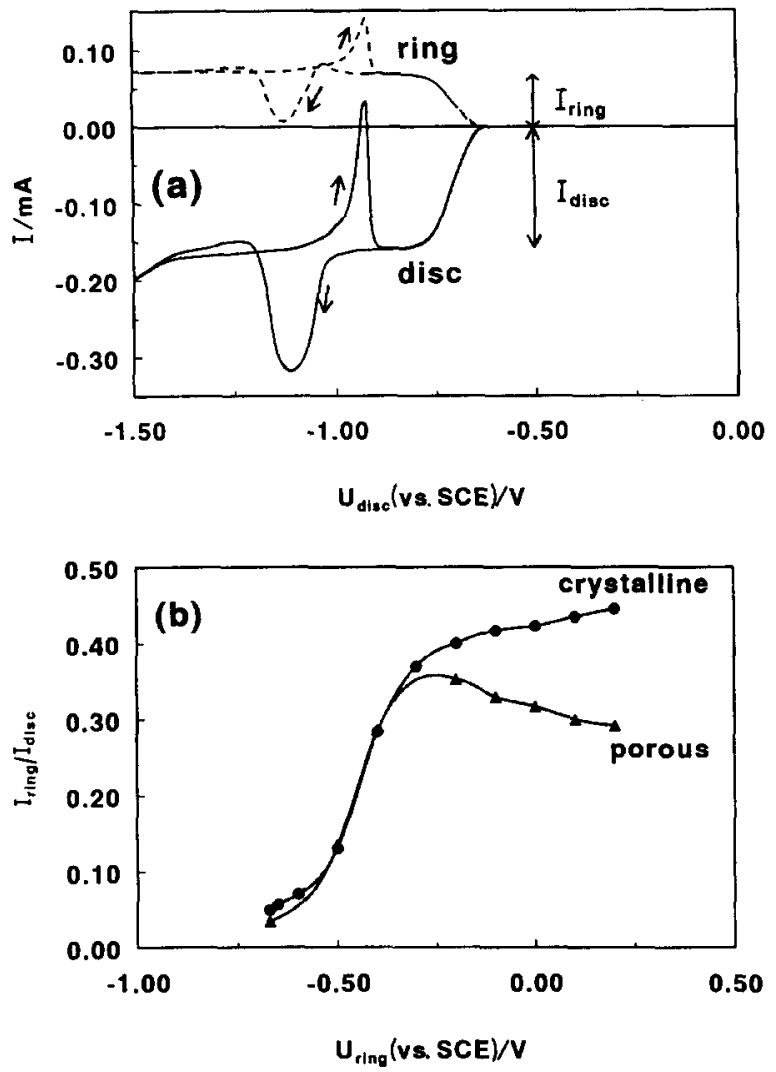

Fig. 5. (a) Potentiodynamic measurement using a dark p-type crystalline silicon-Au RRDE at a fixed ring potential $\left(U_{\text {ring }}=-0.2 \mathrm{~V}\right)$ in $2 \mathrm{mM}$ $\mathrm{MVCl}_{2}+0.1 \mathrm{M} \mathrm{KCl}$ aqueous solution. The disc potential was scanned at $10 \mathrm{mV} \mathrm{s}^{-1}$, while the electrode was rotated at $1600 \mathrm{rev} \mathrm{min}^{-1}$. (b) The potential dependence of the ring current $I_{\text {ring }}$ of p-type crystalline (circles) and porous (triangles) silicon electrodes, normalized with respect to the change in the disc current $I_{\text {disc }}$. Conditions as for Fig. 5(a).

at the ring due to consumption of $\mathrm{MV}^{+\cdot}$ in the formation of $\mathrm{MV}^{0}$ at the disc. The $\mathrm{MV}^{0}$ forms a solid layer at the disc and cannot be detected at the ring. After the peak, the current densities at the ring and the disc return to their initial plateau values. Hydrogen evolution at the disc does not result in a change of the ring current. When the $\mathrm{MV}^{0}$ layer is oxidized in the return scan towards positive potentials, a surplus of $\mathrm{MV}^{+\cdot}$ leads to an increase of the ring current.

For both n-type and p-type silicon, the ring current depends on the potential applied to the ring. The ring current $I_{\text {ring }}$, observed when the disc potential is scanned from the zero current region to the diffusion limited region, is shown in Fig. 5(b) for p-type crystalline (circles) and p-type porous silicon electrodes (triangles) for various values of the ring potential up to $0.2 \mathrm{~V} ; l_{\text {ring }}$ is normalized with respect to the disc current $I_{\text {disc }}$ (see Fig. 5(a)). Oxidation of $\mathrm{MV}^{+} \cdot$ begins at $-0.6 \mathrm{~V}$ with both crystalline and porous electrodes. At more positive potentials $I_{\text {ring }} / I_{\text {disc }}$ for crystalline electrodes reaches a limiting value of 0.46 , which agrees rather well with the calculated collection efficiency of 0.49 . With porous electrodes a maximum is

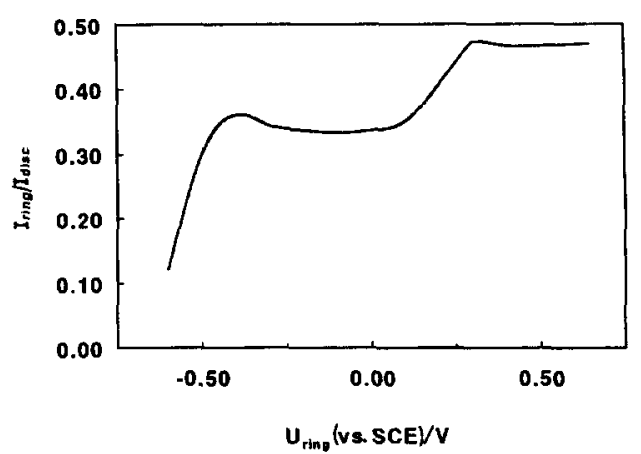

Fig. 6. Current-potential curve of a dark p-type porous silicon ring in 2 $\mathrm{mM} \mathrm{MVCl} \mathrm{MV}_{2}+0.1 \mathrm{M} \mathrm{KCl}$ aqueous solution. The disc potential was kept at a constant value $U_{\text {disc }}=-0.85 \mathrm{~V}$, while the ring potential was scanned at $10 \mathrm{mV} \mathrm{s}^{-1}$ and the electrode was rotated at $1600 \mathrm{rev} \mathrm{min}-1$. The ring current $I_{\text {ring }}$ is normalized with respect to the (constant) disc current $I_{\text {disc }}$.

observed at $-0.3 \mathrm{~V}$, after which $I_{\text {ring }} / I_{\text {disc }}$ decreases to a constant value of approximately 0.30 .

In the experiment of Fig. 6 we scanned the potential of a p-type porous silicon ring up to $0.7 \mathrm{~V}$, while $\mathrm{MV}^{+\cdot}$ was generated at the disc at a constant disc potential $\left(U_{\text {disc }}=\right.$ $-0.85 \mathrm{~V}$ ); the ring current, normalized with respect to the disc current, is plotted as a function of the ring potential. Oxidation of $\mathrm{MV}^{+\cdot}$ again starts at $-0.6 \mathrm{~V}$. After a maximum at $-0.4 \mathrm{~V}$ the current decreases to a value of 0.30 , similar to the results of Fig. $5(\mathrm{~b})$. At $0.2 \mathrm{~V}$ an increase of the ring current to a value of 0.47 is observed; this value corresponds to the collection efficiency of the RRDE. At potentials more positive than $0.8 \mathrm{~V}$ the current increases further due to the anodic oxidation of the porous silicon layer (not shown in Fig. 6) [15].

During the measurements with p-type porous silicon electrodes in $2 \mathrm{mM} \mathrm{MVCl}{ }_{2}$ aqueous solution, no EL due to $\mathrm{MV}^{+\cdot}$ oxidation could be detected. To obtain detectable EL the methylviologen concentration was increased to 0.1 $\mathbf{M}[9]$. In this solution the current-potential characteristics are similar to those of Fig. 6, except that current densities at the ring and at the disc are larger. The EL onset at $0.2 \mathrm{~V}$

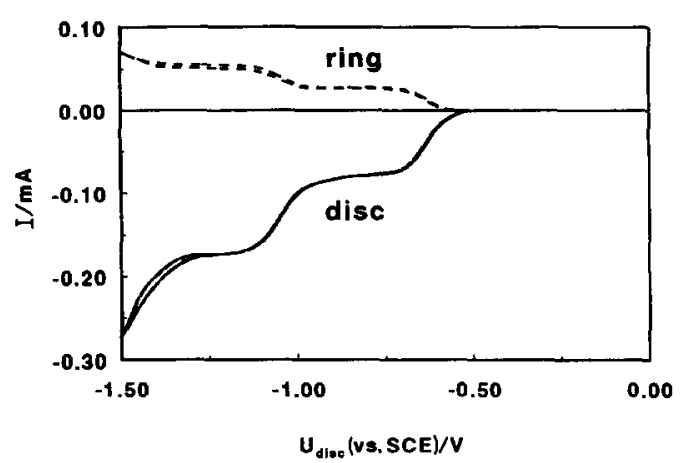

Fig. 7. Potentiodynamic measurement using a p-type crystalline siliconAu RRDE at a fixed ring potential $\left(U_{\text {ring }}=-0.2 \mathrm{~V}\right)$ in $2 \mathrm{mM} \mathrm{MVCl}_{2}+0.1$ $\mathrm{M} \mathrm{KCl} \mathrm{1:1} \mathrm{water} \mathrm{+} \mathrm{ethanol} \mathrm{solution.} \mathrm{The} \mathrm{disc} \mathrm{potential} \mathrm{was} \mathrm{scanned} \mathrm{at} 10$ $\mathrm{mV} \mathrm{s}{ }^{-1}$, while the electrode was rotated at $1600 \mathrm{rev} \mathrm{min}^{-1}$. 
[9] corresponds to the potential at which the increase of the ring current is found in Fig. 6.

Fig. 7 shows the measurements using a p-type crystalline silicon-Au RRDE in $2 \mathrm{mM} \mathrm{MVCl}_{2}+0.1 \mathrm{M} \mathrm{KCl}$ water + ethanol solution. The current-potential curve is different from that in aqueous solution (Fig. 5(a)), because of the higher solubility of the $\mathrm{MV}^{0}$. The anodic ring current is proportional to the disc current in the whole range over which the disc potential is scanned. In the first plateau the $\mathrm{MV}^{+}$. formed at the disc is reoxidized at the ring, as in the RRDE measurements in solutions without ethanol. The $\mathrm{MV}^{0}$ formed in the second plateau does not form a solid layer at the disc, but can be detected at the ring causing a higher ring current.

Measurements with p-type porous silicon ring electrodes in water + ethanol solution yield results similar to those of Fig. 7. We also studied the current-potential characteristics of the porous silicon ring electrode, while generating $\mathrm{MV}^{+\cdot}$ at the disc at a constant potential. A curve similar to that shown in Fig. 6 is obtained; the oxidation of $\mathrm{MV}^{+\cdot}$ starts at $-0.6 \mathrm{~V}$, but now the increase of the current to a value corresponding to the collection efficiency is found at $-0.2 \mathrm{~V}$. Again, no EL was detected in $2 \mathrm{mM} \mathrm{MVCl}_{2}$ water + ethanol solution. Increasing the methylviologen concentration to $20 \mathrm{mM}$ yielded only very weak EL starting at $-0.2 \mathrm{~V}$ with a maximum at about $-0.1 \mathrm{~V}$. Higher concentrations did not lead to an increase of the emission intensity.

\subsection{Capacitance measurements}

To determine the position of the band edges of silicon with respect to the energy levels in solution, we performed Mott-Schottky (M-S) measurements. According to the $\mathrm{M}-\mathrm{S}$ relation for an n-type semiconductor

$C_{\mathrm{sc}}^{-2}=\frac{2}{e \varepsilon_{\mathrm{r}} \varepsilon_{0} N_{\mathrm{D}}}\left(U-U_{\mathrm{fb}}-\frac{k T}{e}\right)$

the squared reciprocal space-charge capacitance per unit area $C_{\text {sc }}$ is a linear function of the applied potential $U$. Typical M-S plots are shown in Figs. 8(a) and 8(b) for $\mathrm{n}$ and p-type crystalline silicon respectively. No significant difference was found between results obtained in an indifferent electrolyte or in solutions containing either $\mathrm{MV}^{2+}$ or $\mathrm{MV}^{+*}$. From the slope of the linear fits we calculate donor and acceptor densities $N_{\mathrm{D}}=4.7 \times 10^{15} \mathrm{~cm}^{-3}$ and $N_{\mathrm{A}}=1.06 \times 10^{15} \mathrm{~cm}^{-3}$. These values are in agreement with the resistivity values given by the supplier of the wafers.

The flat band potential $U_{\mathrm{fb}}$ is obtained by extrapolating the $C_{\mathrm{sc}}^{-2} / U$ plot to $C_{\mathrm{sc}}^{-2}=0$. For $\mathrm{n}$-type crystalline silicon we find $U_{\mathrm{fb}}^{\mathrm{n}}=-0.54 \mathrm{~V}$. Taking into account the difference between the Fermi level $E_{\mathrm{F}}$ and the conduction band edge $E_{\mathrm{CB}}$, we obtain $E_{\mathrm{CB}}=0.77 \mathrm{eV}$. In a similar way we
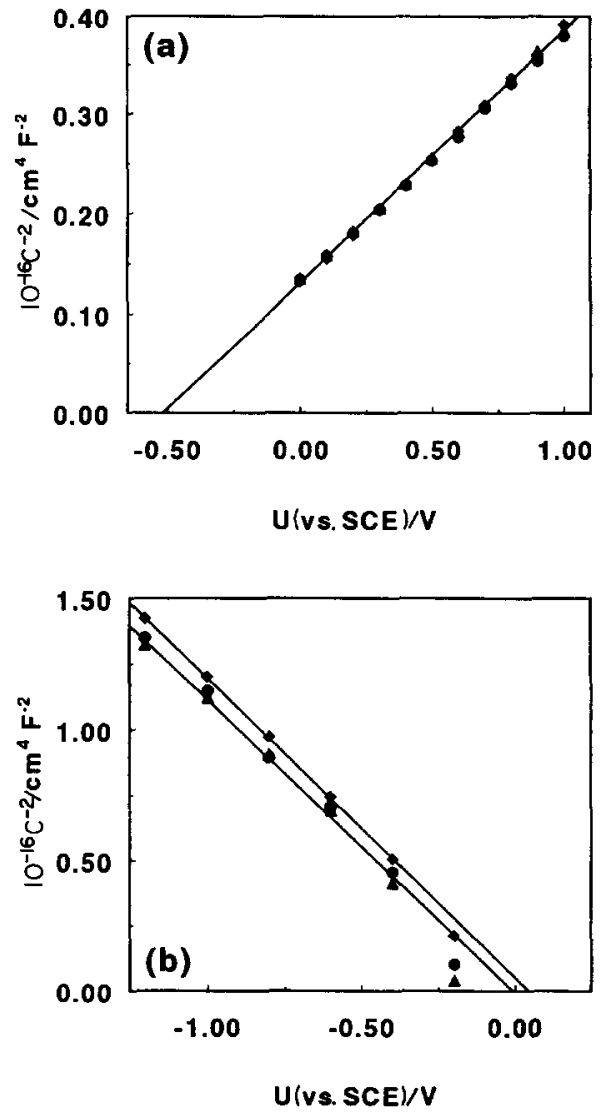

Fig. 8. M-S plots of (a) n-type and (b) p-type crystalline silicon in $2 \mathrm{mM}$ $\mathrm{MVCl}_{2}+0.1 \mathrm{M} \mathrm{KCl}$ aqueous solution. Measuring frequencies were 2.2 $\mathrm{kHz}$ (triangles), $7.0 \mathrm{kHz}$ (circles) and $22.1 \mathrm{kHz}$ (diamonds).

can determine the position of the valence band edge $E_{\mathrm{VB}}$ of p-type silicon. With $U_{\mathrm{fb}}^{\mathrm{p}}=0.06 \mathrm{~V}$, we find $E_{\mathrm{VB}}=-0.30$ $\mathrm{eV}$. The difference $E_{\mathrm{CB}}-E_{\mathrm{VB}}=1.07 \mathrm{eV}$ is close to 1.1 $\mathrm{eV}$, the bandgap of crystalline silicon. Our results agree with those found by Madou et al. [20]. Owing to the similarity between measurements performed in solutions with and without methylviologen, it may be concluded that the presence of methylviologen has little or no effect on the position of the band edges under depletion conditions.

Measurements on porous silicon electrodes were much less reproducible. The $\mathrm{M}-\mathrm{S}$ plots of the n-type material were linear, but often shifted over a few hundred $m V$ towards positive potentials. The slope was the same as that of the crystalline electrode, which indicates that the $C-U$ characteristics are still determined by the bulk semiconductor. The slope of the M-S plots of p-type porous electrodes were also unchanged, but the shift towards positive potentials was much larger, often of the order of $1 \mathrm{~V}$. It is not clear what causes the considerable shift of the flat band potential of porous silicon. Despite the irreproducibility of the impedance measurements, the current-potential characteristics of porous silicon electrodes (as shown in Figs. 3, 5 and 6) were very reproducible and indicate a fixed position of the band edges. 


\section{Discussion}

\subsection{Crystalline silicon}

From impedance measurements it is clear that the difference between $E_{\mathrm{CB}}$, the conduction band edge of n-type, and $E_{\mathrm{VB}}$, the valence band edge of p-type crystalline silicon, is close to the semiconductor band gap; from this we conclude that the band edges of both materials are at the same energy. This leads to the band diagram of Fig. 9 (a). From the current-potential curves in Figs. 1 and 2 we concluded that both reduction reactions of methylviologen occur via electron transfer from the conduction band to the species in solution. The oxidation of $\mathrm{MV}^{0}$ and $\mathrm{MV}^{+\cdot}$ at n-type crystalline electrodes is also a conduction band process, while at p-type material no conclusions can be drawn. However, considering the location of the band edges at both $\mathrm{n}$ - and p-type silicon, it is likely that the oxidation reactions at p-type silicon also take place by electron injection into the conduction band.

With the above in mind, we can analyse the results in Figs. 1 and 2 in relation to the measured flat band potentials. At n-type crystalline silicon the reduction current starts at $-0.7 \mathrm{~V}$, i.e. $0.16 \mathrm{~V}$ more negative than the flat band potential of $-0.54 \mathrm{~V}$. This implies accumulation conditions with a downward band bending of $0.16 \mathrm{~V}$. When the band bending is increased, the Fermi level moves above the conduction band edge. As a result, the surface concentration of electrons becomes large and the electrode acts as a quasi-metal. This explains why the
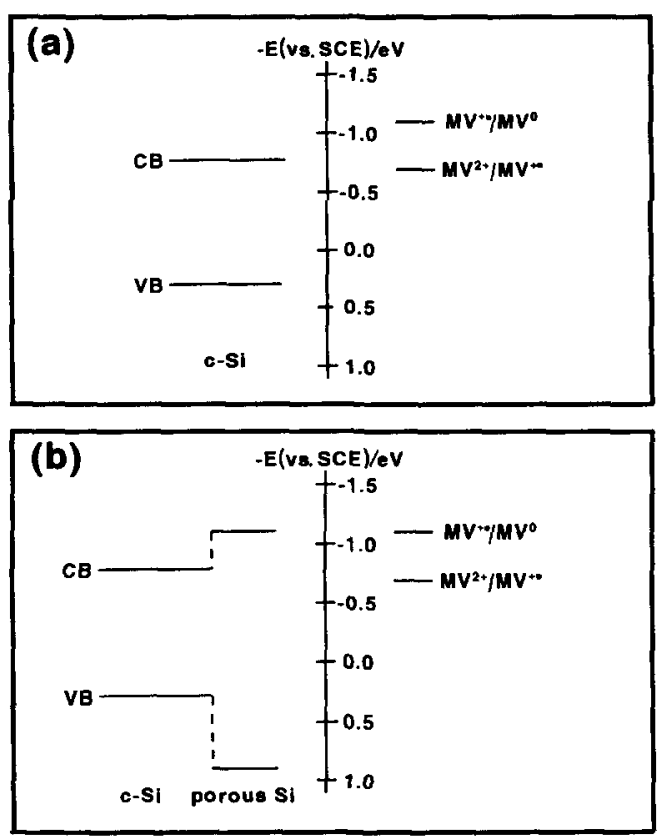

Fig. 9. Schematic energy level diagrams showing the standard redox potentials of the $\mathrm{MV}^{2+}-\mathrm{MV}^{+}$and $\mathrm{MV}^{+\cdot}-\mathrm{MV}^{0}$ redox couples in aqueous solution with respect to the band edges of (a) crystalline and (b) porous silicon. cathodic current at n-type silicon (Fig. 2(a)) strongly resembles the current found at the gold electrode in the ring-disc measurements (Fig. 5(a)). At both types of electrode the first and second reduction reactions start at $-0.69 \mathrm{~V}$ and $-1.05 \mathrm{~V}$ respectively. This is in agreement with the standard redox potentials reported elsewhere $[10,12]$.

For p-type silicon electrodes the situation is different. The reduction current onset at illuminated p-type electrodes (Fig. 1(b)) is at $-0.3 \mathrm{~V}$; at this potential the band bending is about $0.35 \mathrm{~V}$, downwards (depletion conditions). Apparently, a considerable band bending is necessary to prevent recombination via surface states leading to a separation of the photogenerated charge carriers. In the RRDE measurements with p-type crystalline silicon (Fig. 5) electron injection by oxidation of $\mathrm{MV}^{+\cdot}$ is observed at potentials more positive than $-0.6 \mathrm{~V}$. This implies that, despite the unfavourable band bending of $0.65 \mathrm{~V}$, recombination of the injected electrons with holes takes place.

\subsection{Porous silicon}

Although the flat band potentials obtained from the impedance of porous silicon electrodes are undependable, we can compare the current-potential characteristics of crystalline and porous silicon. The electrolyte penetrates into the porous structure and forms a contact to both the crystalline substrate at the pore fronts and the porous semiconductor. The question arises as to whether electron transfer occurs at the bottom of the pores or within the porous layer. Current-potential curves of crystalline and porous silicon are generally similar, as long as $\mathrm{MV}^{0}$ is not formed at the porous electrodes. The onset of $\mathrm{MV}^{2+}$ reduction in Figs. 1(a) and 3 occurs at approximately the same potential. The same is observed in Figs. 5(b) and 6 for the oxidation of $\mathrm{MV}^{+\cdot}$ at crystalline and porous silicon; at both types of electrode the current onset is found at $-0.6 \mathrm{~V}$. We assume that this similarity is due to the fact that electron transfer takes place at the pore fronts; the $\mathrm{MV}^{+\cdot}$ injects electrons into the conduction band of the crystalline substrate. No EL is detected between $-0.6 \mathrm{~V}$ and $0.2 \mathrm{~V}$, which also supports the above view. From this we conclude that the position of the band edges of crystalline silicon are the same in the unetched and porous etched electrodes. If we then take into account the larger band gap of the porous semiconductor and the mismatch of the band edges at the silicon/porous silicon junction $(70 \%$ and $30 \%$ at the valence band and the conduction band respectively $[21,22])$, we obtain a schematic energy level diagram for the silicon/porous silicon structure as shown in Fig. 9(b). This diagram indicates that, under normal conditions, $\mathrm{MV}^{+\cdot}$ should be able to inject electrons into the conduction band of crystalline silicon, but not into the porous structure.

Two plateaus are found in Fig. 6. As mentioned above, the first plateau is due to electron injection into the crys- 
talline substrate. When the potential is scanned towards more positive values an increase of the anodic current is observed at $0.2 \mathrm{~V}$. This potential corresponds to the onset of EL [9]. In ethanol solutions the current-potential curves are similar to those of Fig. 6 , but the second increase is now found at $-0.2 \mathrm{~V}$. This potential again corresponds to the onset of EL in these solutions. This indicates that the current in the second plateau is due at least partly to electron injection into the porous silicon structure.

With this in mind we can speculate on the physical background for the observed current-potential and ELpotential characteristics. The current onset at $-0.6 \mathrm{~V}$ is due to electron injection into the crystalline substrate at the bottom of the pores. A small part of the electrons may be injected into the porous layer but no recombination takes place as the hole concentration is very low due to the downward band bending (depletion conditions) and the rather large valence band edge mismatch. If we assume that the position of the band edges initially remains unchanged, the band bending in the substrate decreases when the potential is scanned towards positive values. A decrease of the band bending leads to a higher concentration of holes at the silicon/porous silicon interface, which implies that the hole concentration in the porous structure also increases. When the flat band potential is approached, deviations from the linear M-S plot are found (Fig. 8(b)). At potentials more positive than the flat band value, part of the applied potential will appear over the Helmholtz layer and the semiconductor band edges will shift to lower energy. Electron injection into the porous structure now becomes possible, thereby accounting for the increase of the current at $0.2 \mathrm{~V}$ in Fig. 6. In more concentrated methylviologen solution the injection current due to $\mathrm{MV}^{+}$. oxidation is larger, and part of the recombination is radiative which results in the observed EL [9].

\section{Conclusions}

Using stationary and rotating disc and rotating siliconring gold-disc electrodes, we have studied the electrochemistry of methylviologen at crystalline and porous silicon. It was concluded that the two reduction reactions of methylviologen proceed by electron capture from the conduction band of both n- and p-type crystalline and porous silicon. The oxidation reactions at n-type electrodes occur via electron injection into the conduction band. On the basis of capacitance measurements, we conclude that this is also the case at p-type crystalline silicon. At p-type porous silicon, injection of electrons by $\mathrm{MV}^{+} \cdot$ leads to EL in concentrated methylviologen solutions.

In solutions containing ethanol, the current-potential curves are different from those measured in aqueous solu- tion owing to the solubility of $\mathrm{MV}^{0}$, the product of the second reduction step. The EL intensity is not enhanced, as was the case for $n$-type porous silicon.

The differences in the current-potential measurements found with porous silicon are discussed on the basis of a schematic energy level diagram. A downward shift of the band edges at potentials more positive than the flat band value for $\mathrm{p}$-type silicon facilitates electron injection into the porous layer.

\section{Acknowledgements}

The authors would like to thank G.H. Schoenmakers and $\mathrm{R}$. Waagenaar for helpful discussions. The work described here was supported by the Netherlands Foundation for Chemical Research (SON) with financial aid from the Netherlands Organization for Scientific Research (NWO).

\section{References}

[1] L.T. Canham, Appl. Phys. Lett., 57 (1990) 1046.

[2] N. Koshida and H. Koyama, Appl. Phys. Lett., 60 (1992) 347.

[3] F. Namavar, H.P. Maruska and N.M. Kalkhoran, Appl. Phys. Lett., 60 (1992) 2514.

[4] F. Kozlowski and W. Lang, J. Appl. Phys., 72 (1992) 5401.

[5] P.M.M.C. Bressers, J.W.J. Knapen, E.A. Meulenkamp and J.J. Kelly, Appl. Phys. Lett., 61 (1992) 108.

[6] L.T. Canham, W.Y. Leong, M.I.J. Beale, T.I. Cox and L. Taylor, Appl. Phys. Lett., 61 (1992) 2563.

[7] E.A. Meulenkamp, P.M.M.C. Bressers and J.J. Kelly, Appl. Surf. Sci., 64 (1993) 283.

[8] A. Bsiesy, F. Muller, M. Ligeon, F. Gaspard, R. Herino, R. Romestain and J.C. Vial, Phys. Rev. Lett., 71 (1993) 637.

[9] E.S. Kooij, R.W. Despo and J.J. Kelly, Appl. Phys. Lett., 66 (1995) 2552.

[10] C.L. Bird and A.T. Kuhn, Chem. Soc. Rev., 10 (1981) 49.

[11] M. Heyrovský, J. Chem. Soc. Chem. Commun., (1987) 1856.

[12] D.W. Bahnemann, C.H. Fischer, E. Janata and A. Henglein, J. Chem. Soc. Faraday Trans 1, 83 (1987) 2559.

[13] P.A. Christensen and A. Hamnett, J. Electroanal. Chem., 263 (1989) 49.

[14] K. Ogasawara, T. Momma and T. Osaka, Chem. Lett., (1994) 1243.

[15] A. Halimaoui, Appl. Phys. Lett., 63 (1993) 1264.

[16] K. Ogasawara, T. Momma and T. Osaka, J. Electrochem. Soc., 142 (1995) 1874.

[17] W.J. Albery and M.L. Hitchman, Ring-Disc Electrodes, Clarendon, Oxford, 1971.

[18] CRC Handbook of Chemistry and Physics, CRC, Boca Raton, FL, 1986, 66th edn.

[19] A.J. Bard and L.R. Faulkner, Electrochemical Methods, Wiley, New York, 1980.

[20] M.J. Madou, B.H. Loo, K.W. Frese and S.R. Morrison, Surf. Sci., $108(1981) 13$.

[21] T. van Buuren, T. Tiedje, J.R. Dahn and B.M. Way, Appl. Phys. Lett., 63 (1993) 2911.

[22] P.H. Hao, X.Y. Hou, F.L. Zhang and X. Wang, Appl. Phys. Lett., 64 (1994) 3602. 